\title{
Influencing Agent Group Behavior by Adjusting Cultural Trait Values
}

\author{
Gaurav Tuli and Henry Hexmoor, Senior Member, IEEE
}

\begin{abstract}
Social reasoning and norms among individuals that share cultural traits are largely fashioned by those traits. We have explored predominant sociological and cultural traits. We offer a methodology for parametrically adjusting relevant traits. This exploratory study heralds a capability to deliberately tune cultural group traits in order to produce a desired group behavior. To validate our methodology, we implemented a prototypical-agentbased simulated test bed for demonstrating an exemplar from intelligence, surveillance, and reconnaissance scenario. A group of simulated agents traverses a hostile territory while a user adjusts their cultural group trait settings. Group and individual utilities are dynamically observed against parametric values for the selected traits. Uncertainty avoidance index and individualism are the cultural traits we examined in depth. Upon the user's training of the correspondence between cultural values and system utilities, users deliberately produce the desired system utilities by issuing changes to trait. Specific cultural traits are without meaning outside of their context. Efficacy and timely application of traits in a given context do yield desirable results. This paper heralds a path for the control of large systems via parametric cultural adjustments.
\end{abstract}

Index Terms-Command and control, intelligence, surveillance and reconnaissance (ISR), man on the loop, multiagent system, supervisory control, unmanned vehicles.

\section{NOMENCLATURE}

$\begin{array}{ll}\text { C2 } & \text { Command and control. } \\ \text { CLV } & \text { Collectivism. } \\ \text { CRU } & \text { Capability resource unit. } \\ \text { DOD } & \text { Department of Defense. } \\ \text { ISR } & \text { Intelligence, surveillance, and reconnaissance. } \\ \text { IAS } & \text { ISR agent society. } \\ \text { IDV } & \text { Individualism. } \\ \text { LTO } & \text { Long-term orientation. } \\ \text { MOTL } & \text { Man on the loop. } \\ \text { NCW } & \text { Network centric warfare. } \\ \text { PDI } & \text { Power Distance Index. } \\ \text { PL } & \text { Preference list. } \\ \text { UAI } & \text { Uncertainty Avoidance Index. }\end{array}$

Manuscript received January 3, 2009; revised July 14, 2009 and October 13, 2009. This work was supported by the Air Force Research Laboratory under Contract FA8750-06-C-0138. This paper was recommended by Associate Editor J. Liu.

G. Tuli was with the Department of Computer Science, Southern Illinois University, Carbondale, IL 62901 USA. He is now with the Computer Science Department, Virginia Polytechnic Institute and State University, Blacksburg, VA 24061 USA (e-mail: gtuli@cs.siu.edu).

H. Hexmoor is with the Department of Computer Science, Southern Illinois University, Carbondale, IL 62901 USA (e-mail: hexmoor@cs.siu.edu).

Color versions of one or more of the figures in this paper are available online at http://ieeexplore.ieee.org.

Digital Object Identifier 10.1109/TSMCB.2009.2036592

\author{
UAV Unmanned aerial vehicle. \\ ULD UAI level deciding. \\ USAF-SAB United States Air Force Scientific Advisory \\ Board.
}

\section{INTRODUCTION}

$\mathcal{C}$ ORPORATE or organizational culture is a notion that is often considered to be nebulous and soft. Although it is commonly accepted to have a pivotal effect in performance, contemporary formulations lack rigor to enable construction of instrumental tools. Albeit in nascence, we start from the basic cultural dimensions and then take steps toward rigors of specifying cultural parameters for groups. Cultural parameters are fundamental engineering instruments for monitoring and directing culture in large groups. We will not be concerned with directly managing values and norms. By global changes in reasoning of how individuals relate to one another, collective behaviors are indirectly produced. We will examine the dynamics of organizational culture in the military context of $\mathrm{C} 2$.

The NCW paradigm initiated by the DOD prescribes requirements for the information sharing and human-automated system collaboration [4]. Operators are expected to leverage multiple information sources for decisions under significant time pressure and when there is uncertainty. However, an increase in the available information resources will place higher cognitive demands on the operators, which could become constraints that limit the success of network centric processes. Therefore, NCW advocates the socialization of (military) action through the socialization of each intent, capability, and awareness [5].

The DOD roadmap for unmanned aircraft system development has also predicted that future UAVs will work as independent robots, which can self-actualize to perform a given task instead of just working as teleoperated robots [6]. To lift the current unmanned vehicles (UVs) to this level, we selected the most widely explored cultural parameters given by social scientists, notably by Hofstede [10]. These parameters will socialize both military forces and warfare machinery, which provides leverage for performing high-level decision making to a human supervisory body as presented in the MOTL paradigm [2], [4], [8].

We are exploring the applicability of the cultural parameters in complex control systems, like ISR system, to make high-level human supervision possible. In order to model the dynamic nature of ISR-C2, the reasoning capabilities of the agents or ISR agents have been used in this paper as the key attribute, which distinguishes among agents. An agent is any software entity with predefined capabilities that can achieve a goal. 
After following the recommendations given by several federal agencies, such as the DOD and the USAF-SAB, we have tailored our approach to promote the recommended changes in the present automation level of human supervisory control. The utilitarian approach has been utilized herein to create a few norms and rules for implementing the cultural parameters in a nonhuman cultural setting [7]. Moreover, special care has been given to design Pareto optimal ISR agents, which may sacrifice their personal benefits in favor of social benefits [18].

The paper is organized as follows. The next section outlines the details of MOTL and selected cultural parameters. Section III delineates our approach of socializing the agent community by forming and imposing laws using utility theory and role exchange, respectively, and finally presents our algorithm. Section IV presents the implementation of the algorithm in the form of simulated agent-based experiments, revealing several facets of our approach. In Section V, we present the results and conclusions of this paper. Lastly, Section VI includes the future research possibilities based on this work.

\section{MOTL AND Its Cultural PARAmeters}

In the shadow of demanding technological advancements in the field of defense like the NCW, many federal scientific agencies, including the USAF-SAB and DOD, have clearly urged the need for improvements in supervisory control [6], [23]. The level of human intervention for supervision can be broadly divided into two categories. If a human interference directly affects actions of a specific acting entity (i.e., an agent) in the control loop, it was traditionally termed as Man In The Loop (or MITL). On the other hand, if the human intervention indirectly affects actions at the unit or agent community level, it was coined as MOTL [8].

MOTL is a socio-psycho-cultural model to observe and mediate behaviors and interactions of complex control systems. It is characterized as a paradigm shift where a human supervisor employs psychological and social influences to control the system behavior. MOTL alleviates excessive dependence on the human supervisor. MOTL by its very nature could be considered as the Sheridan's strict form of supervisory control [20]. A system-level network policy management is an example for MOTL, where changing the policies in the policy management system by the system administrator will affect the configuration of the entire network without tediously altering each system parameter [22].

Hence, MOTL presents a foundation to a novel high-level human supervision that contrasts with typical micromanagement of tasks in complex control systems where large collections of communities are usually founded. Such complex control systems are customary in the NCW due to the notable changes in several war fighting trends. The shift in warfare paradigm from platform centric to network centric has significantly changed the entire human supervision role, both in mission planning and actual operations, as shown by Lambert and Scholz using a real-time example [14, pp. 6]. Instead of controlling the system manually, now, military operators are more involved in the higher levels of planning and decision making.

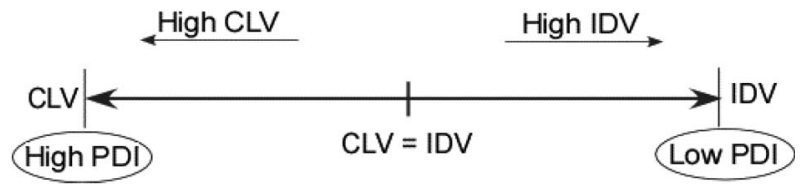

Fig. 1. IDV (or CLV) spectrum and IDV-PDI relationship.

The key attributes of MOTL that characterizes its novelty are based on well-founded theories in social sciences and fall into three main categories, namely, personality, social reasoning, and culture [8]. To avoid cultural differences, which may tend to decrease coordination in an agent society, MOTL uses vital cultural parameters given by Hofstede, namely, UAI, IDV, PDI, LTO, and masculinity [10].

Due to the nature of ISR, particularly during war with people and machine of differing cultures, uncertainty cannot be overlooked, and control over this parameter will eventually improve the whole system. Therefore, we included UAI in our research. It has also been shown that there is a strong interconnection between UAI, IDV, and PDI [1], [9]. Hence, the benefit of including IDV and PDI into control and command has also been explored in this research. However, LTO is worth exploring but is outside of our scope. Similarly, since we are mainly modeling artificial agents and they lack gender characteristics, masculinity was not considered in MOTL. Next, we briefly discuss the selected cultural parameters in more details.

\section{A. UAI}

Situational awareness is often reduced in a warlike scenario due to "Fog of War" that leads to uncertainty [17]. Uncertainty is also an integral characteristic of organizational decision making and any cultural makeup [3]. Hofstede explained uncertainty as the dimension that does not relate to risky situations but rather to unknown or unfamiliar situations [10]. Different cultures have different levels of tolerance for uncertainty and ambiguity that is measured and represented using UAI [10]. UAI indicates the extent to which a culture programs its members to feel either uncomfortable (i.e., high UAI) or comfortable (i.e., low UAI) in an unstructured situation. The level of imposed restrictions defines the respective level of comfort for the member of a particular culture. UAI is usually enforced by strict laws and rules, safety, and security measures. We have defined certain stringent rules in Section III for our agents to restrict their behavior while working with culturally similar or diverse members.

\section{B. IDV}

IDV and CLV describe the relationship between the individual and the collectivity that prevails in a given society, but both reside at the opposite ends, as shown in Fig. 1. The individualistic end of continuum construes a person as a separate entity, which is clearly distinguishable from social milieus [9]. On the other hand, in a collectivist culture, the distinction between the individual and the group becomes blurred, and the people regard themselves as the extension of a social system [13]. In the context of self-theory, the people who are a part of 
more individualist cultures have selves or self-cognitions. The individualistic people refer themselves as independent, selfcontained, autonomous, and distinct units, whereas people in collectivist cultures give more importance to interdependence or sociocentric identity [15].

\section{PDI}

PDI measures the extent to which the less powerful members of a society accept and expect that power to be distributed unequally [10]. This index has been incorporated into MOTL to accommodate inherited or developed inter- and intra-power differences among several working agent communities in a complex system. Hence, PDI will capture the degree of inequality among agents in an agent society and measure how much a society has respect for authority. High PDI ranking indicates that the inequalities of power and social values like autonomy, trust, reliance, and benevolence will be allowed to grow within a group [10]. In such a system, agents may view the supervisor as a benevolent dictator and obey her orders. Conversely, low PDI ranking indicates that the group de-emphasizes the differences between the agent's power and social values [10]. Hence, equality and opportunity for every agent are stressed in this case.

There exists an inherent relationship between IDV and PDI. Bochner and Hesketh found that only collectivist culture can work under pressure [1]. They studied individuals in a culturally diverse work setting and found that those who belong to high collectivist cultural background allow or feel comfortable while working under their boss' supervision. These individuals prefer a boss or a manager to be more autocratic and paternalistic, whereas individuals who belong to high individualistic culture prefer their boss or manager to be more hands-on and consultative [1]. Hence, only high CLV (or low IDV) cultures will allow high PDI. This spectrum of IDV-PDI relationship is also shown in Fig. 1.

\section{Socializing Agents}

Supervisory control paradigm is in need of a paradigm shift to invert the current many-to-one operator to UV ratio. Herein, we present our strides toward this new paradigm by socializing the agents. We replicated individual as agents, group of agents as culture, and group of cultures as society. Let us take an example of ISR operations. An ISR agent, denoted as agent in the literature, is a software component, which takes part in an ISR mission. For example, a software component responsible for the combat behavior, a component responsible for goals setting, or a software component responsible for communication with a ground station can be considered as an agent. Different cultural traits or properties of agents lead to different agent cultures, and a collection of agents with different or similar cultural traits leads to agent society, which we denoted as IAS.

In a multiagent environment, as in IAS, every agent would perform its role in collaborative actions to achieve a welldefined goal. For example, swiftly performing ISR in an adversary's territory with the lowest possible damage to the IAS is a well-defined goal. A C2 personnel or a human supervisor can transform or build such goals and can also supervise the actions of the IAS agents using MOTL. The human supervisor or MOTL operator is addressed as Moe in this paper. Training Moe to identify the correspondence between the agents' cultural values and MOTL-based supervisory tools would improve her efficacy to administer IAS. However, discussion on such training is out of the scope of this paper.

The basic aim of this paper is to make agents of different cultures work collectively in an IAS. Hence, a sense of society is given to the agents that will allow the agents to take socially responsible decisions while working under the same umbrella of dirty, dull, and dangerous tasks. This will lead to the formation of a big IAS holding several small or big agent cultures. These IAS could either be heterogeneous, if one or more agent cultures belong to different cultural settings, or homogeneous, if the cultural settings of all the participating agent cultures are similar [10]. Moreover, similar to most social processes, many agents of an IAS have to bear personal sacrifices to increase social benefits [16]. Our approach mimics this social phenomenon using IAS that obeys the Pareto optimal principle [18].

\section{A. Rule- and Norm-Based UAIs}

In order to limit the inherent uncertainties in ISR, we imposed strict laws and rules over IAS. These rules provide us with capabilities for fine tuning or controlling uncertainty factors. Our paradigm aims the following: 1) reduce uncertainty for collaborative work and 2) make a control task to be less tiresome. To achieve these goals, we have designed our algorithm using concepts from utility theory and joint benefits.

To deal with the multidimensionality of alternatives in a complex decision-making system, we used additive and lexicographic properties while dealing with our agents' utility function [7]. The additive property of the utility function helps to represent an agent as a collection of various attributes, such as its functional capabilities, whereas the lexicographic property would allow an agent to identify more important attributes of the utility function among other attributes. The significance of using these features is explained with an example later in this section.

Similarly, to allow equal distribution of benefits to the participating agent cultures for collaborative achievements, we used the joint-benefit decision-making approaches given by Kalenka and Jennings [12]. The joint-benefit approach is the combination of individual-and social-benefit decision-making approaches. In the individual-benefit-based approach, an agent performing the lead role will always get the biggest piece of the benefit, whereas the social-benefit approach offers a proportional share of the benefits to all team members [12]. Hence, the joint-benefit approach prefers to maximize both the individual and social benefits. In our approach, we considered the social benefit as the summation of social gain and social loss, whereas the individual benefits as the summation of individual gain and individual loss, as given by

Joint Benefit $=$ Individual gain - Individual loss

$$
+ \text { Social gain - Social loss. }
$$




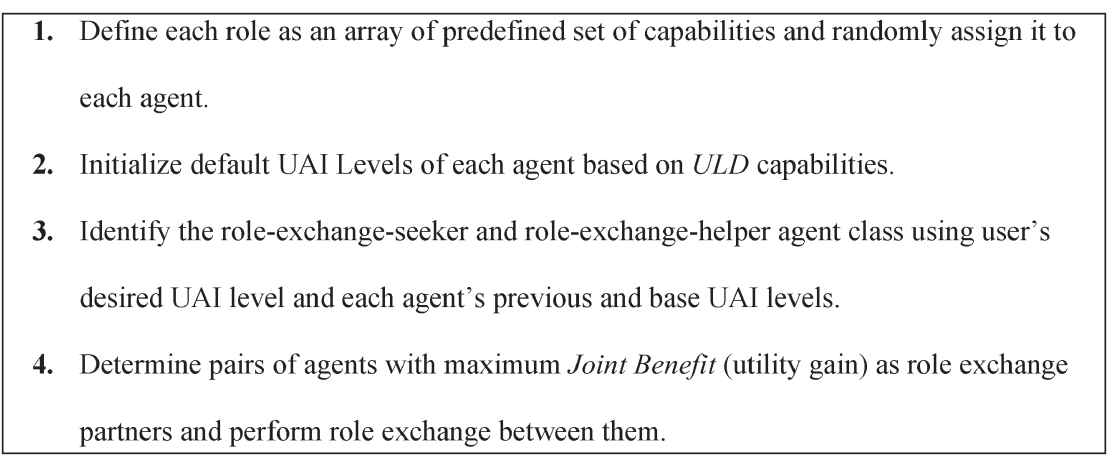

Fig. 2. Algorithm (given at a high level).

\section{B. Implementing Rules and Norms on IAS}

In order to improve the joint benefit, we implemented the UAI in IAS using a role-exchange technique called the utilitybased role exchange [19]. Hence, to achieve a well-defined goal, the agents will exchange their roles based on the jointbenefit value calculated according to (1). The reasoning for role swapping is discussed next.

The first reasoning that an agent requires is to answer the following question: Whom do I ask for a role exchange? The rational negotiation strategies defined by Rahman and Hexmoor are utilized to answer this question [19]. Using the rational approaches of negotiation, an agent will compute the joint benefit with respect to every agent that belongs to its priority list. After computing the utility value for the agents in the priority list, the next dilemma would be to decide: Which utility value should I select for the role exchange? We used the individual optimal role-exchange scheme to answer this question, which searches each agent's priority list and selects a maximum joint-benefit-producing agent as the roleexchange partner [24], [25]. Moreover, to make our approach Pareto optimal, we used such a utility function which will provide us with a socially committed priority list with every agent willing to exchange roles irrespective of its personal losses.

\section{Role-Exchange Algorithm Using Joint Benefits}

Let us now discuss the procedure that utilizes the concepts discussed so far and allows agents to encompass the diversity, dynamicity, and versatility of ISR. A four-step algorithm of this procedure is shown in Fig. 2. Details of the steps are described in text.

1) Agents, Capabilities, and Roles: An IAS is a collection of agents of similar or different cultural traits, where an agent can be represented as $\mathbf{a}_{\boldsymbol{i}}$ and the collection of such agents by set AGENT. The proposed algorithm uses the agents' capabilities to distinguish among agents and among roles performed by these agents. The set $\mathbf{C A P}$ represents the collection of different capabilities $\mathbf{c}_{\boldsymbol{j}}$.

We assume that each of these capabilities is generated by a CRU. Hence, a CRU can be considered as a collection of equipment and machineries, responsible to provide a capability to an agent. For example, an attack CRU of a combat vehicle would be a collection of advanced embedded systems and several lethal weapons, which provides the attack capability to this combat vehicle. Every such physical unit that provides a capability to an agent is considered as a CRU in this paper. Hence, we can say that each capability $\mathbf{c}_{j}$ of an agent $\mathbf{a}_{i}$ is generated by a CRU, denoted as $C R U_{\mathbf{c}_{j}}^{\mathbf{a}_{i}}$. Moreover, these capabilities are generated in a wide spectrum of values, denoted by set $\mathrm{V}_{\mathbf{c}_{j}}^{\mathbf{a}_{i}}$. At any time $\mathbf{t}$, an agent $\mathbf{a}_{\boldsymbol{i}}$ possesses a combination of capabilities, i.e., $\mathbf{c}_{1}, \mathbf{c}_{2}, \ldots, \mathbf{c}_{\boldsymbol{n}}$, and their corresponding values $\mathbf{v}_{\mathbf{c}_{\boldsymbol{1}}, \mathbf{t}}^{\mathbf{a}_{\boldsymbol{i}}}, \mathbf{v}_{\mathbf{c}_{\boldsymbol{2}}, \mathbf{t}}^{\mathbf{a}_{\boldsymbol{i}}}, \ldots, \mathbf{v}_{\mathbf{c}_{n}, \mathbf{t}}^{\mathbf{a}_{i}}$, where $1 \leq \boldsymbol{n} \leq$ $|\mathbf{C A P}|$ and $\mathbf{v}_{\mathbf{c}_{j}, \mathbf{t}}^{\mathbf{a}_{i}} \in \mathrm{V}_{\mathbf{c}_{j}}^{\mathbf{a}_{i}} .|\mathbf{C A P}|$ denotes the total number of capabilities in an IAS, and value $\mathbf{v}_{\mathbf{c}_{j}, \mathbf{t}}^{\mathbf{a}_{\boldsymbol{i}}}$ denotes the value of a capability $\mathbf{c}_{\boldsymbol{j}}$ that an agent $\mathbf{a}_{\boldsymbol{i}}$ is using at time $\mathbf{t}$, where $0 \leq$ $\mathbf{v}_{\mathbf{c}_{j}, \mathbf{t}}^{\mathbf{a}_{i}} \leq \operatorname{MaxCap_{\mathbf {c}_{j}}} \mathbf{a}_{\mathbf{a}_{i}}$ MaxCap $\mathbf{c}_{\mathbf{c}_{j}}^{\mathbf{a}_{i}}$ represents the maximum value of capability $\mathbf{c}_{j}$, which a $C R U_{\mathbf{c}_{j}}^{\mathbf{a}_{i}}$ can generate for an agent $\mathbf{a}_{\boldsymbol{i}}$.

During a mission endeavor, each agent is required to perform some role(s). In an IAS, a role can be represented as $\mathbf{r}_{k}$ and the collection of such roles by set ROLE. To perform a role fully, an agent requires a set of capabilities with certain value. For example, at any time $\mathbf{t}$, role $\mathbf{r}_{\boldsymbol{k}}$ can be fully performed by agent $\mathbf{a}_{\boldsymbol{i}}$, only if $\mathbf{a}_{\boldsymbol{i}}$ possesses the capabilities $\mathbf{c}_{\mathbf{1}}, \mathbf{c}_{\mathbf{2}}, \ldots, \mathbf{c}_{\boldsymbol{n}}$ and uses them at the required value levels $\mathbf{v}_{\mathbf{c}_{1}, \mathbf{t}}^{\mathbf{r}_{k}}, \mathbf{v}_{\mathbf{c}_{2}, \mathbf{t}}^{\mathbf{r}_{k}}, \ldots, \mathbf{v}_{\mathbf{c}_{n}, \mathbf{t}}^{\mathbf{r}_{k}}$, respectively. Each of the $\mathbf{v}_{\mathbf{c}_{j}, \mathbf{t}}^{\mathbf{r}_{k}}$ denotes the required value for a capability $\mathbf{c}_{j}$ to perform a role $\mathbf{r}_{\boldsymbol{k}}$. This implies that, if $\mathbf{v}_{\mathbf{c}_{j}, \mathbf{t}}^{\mathbf{r}_{k}} \leq \mathbf{v}_{\mathbf{c}_{j}, \mathbf{t}}^{\mathbf{a}_{i}}$, then $\mathbf{a}_{\boldsymbol{i}}$ could performs the role $\mathbf{r}_{\boldsymbol{k}}$ fully using value $\mathbf{v}_{\mathbf{c}_{j}, \mathbf{t}}^{\mathbf{a}_{i}}$ at $\mathbf{v}_{\mathbf{c}_{j}, \mathbf{t}}^{\mathbf{r}_{k}}$; otherwise, it performs the role partially using a value below $\mathbf{v}_{\mathbf{c}_{j}, \mathbf{t}}^{\mathbf{r}_{k}}$. Also, one point to be noted that, with the changing requirements of an ISR mission, both the agent $\mathbf{a}_{\boldsymbol{i}}$ 's role assignments and the required value of a capability $\mathbf{c}_{\boldsymbol{j}}$ to perform a role $\mathbf{r}_{k}$ or $\mathbf{v}_{\mathbf{c}_{j}, \mathbf{t}}^{\mathbf{r}_{k}}$ can change.

In an IAS, the number of roles assigned to an agent depends upon the mapping between the total number of agents or AGENT $\mid$ and the total number of roles to be performed or $|\mathbf{R O L E}|$. If $|\mathbf{A G E N T}|=|\mathbf{R O L E}|$, then there will be a one-to-one mapping, and one agent will perform one role at any given time t. Similarly, if $\mid$ AGENT $|<|$ ROLE $\mid$, then more than one role would be assigned to an agent, and if $\mid$ AGENT $|>|$ ROLE $\mid$, then more than one agent will pursue a role. However, in order to avoid any complex situation that may reduce the clarity of this research, we restricted our experiments discussed in Section IV just to use $|\mathbf{A G E N T}|=$ |ROLE $\mid$.

2) ULD Capabilities: There is a relationship between military operations such as attack and perceptions of unfolding 
events. The skills or capabilities used in such military operations entail ambiguity and unfamiliarity into the system. We call this special class of capabilities as the ULD capabilities, denoted by set $\mathbf{U C A P}$, where $\mathbf{U C A P} \subseteq \mathbf{C A P}$. This implies that set CAP is a collection of non-ULD and ULD capabilities. Moreover, as these uncertainty-inducing capabilities are the integral parts of an ISR operation, therefore, UCAP $\neq \varnothing$ in an IAS.

The level of uncertainty induced in an IAS depends upon the values at which the ULD capabilities are being used by its agents to perform their roles. Suppose that $U A I_{\mathrm{t}}^{\mathbf{a}_{i}}$ is the tolerance value for uncertainty and ambiguity (or UAI) that an agent $\mathbf{a}_{i}$ satisfies at time $\mathbf{t}$. Therefore, $U A I_{\mathbf{t}}^{\mathbf{a} i}$ depends upon the value of each of the ULD capability, denoted as $\mathbf{c}_{l}$, used by $\mathbf{a}_{i}$ to perform a role $\mathbf{r}_{k}$ or $\mathbf{v}_{\mathbf{c}_{l}, \mathbf{t}}^{\mathbf{a}_{i}}$. Moreover, the bigger this value, the lower the $U A I_{\mathbf{t}}^{\mathbf{a}_{i}}$. Hence, $U A I_{\mathbf{t}}^{\mathbf{a}_{i}}$ can be calculated as the inverse function of the summation of all $\mathbf{v}_{\mathbf{c}_{l}, \mathbf{t}}^{\mathbf{a}_{i}}$, as shown in

$$
U A I_{\mathbf{t}}^{\mathbf{a}_{i}}=f\left(\frac{1}{\sum_{l=1}^{|U C A P|} \mathbf{v}_{\mathbf{c}_{l}, \mathbf{t}}^{\mathbf{a}_{i}}}\right) .
$$

3) Role Exchange in IAS: An IAS comprises of agents from different cultures with different UAIs. As per the UAI definition explained in Section II, an agent culture with the lowest UAI value dictates the UAI of an IAS or $U A I_{\mathbf{t}}^{\mathrm{IAS}}$. However, (2) implies that changing $\mathbf{v}_{\mathbf{c}_{l}, \mathbf{t}}^{\mathbf{a}_{i}}$ would change the $U A I_{\mathbf{t}}^{\mathbf{a} i}$, and if $U A I_{\mathrm{t}}^{\mathbf{a} i}$ is altered for every agent $\mathbf{a}_{\boldsymbol{i}}$, then eventually, we can change the $U A I_{\mathbf{t}}^{\mathrm{IAS}}$. Moe can enforce a UAI value, denoted by $U A I_{\mathbf{t}}^{\mathrm{Moe}}$, to collectively change an IAS behavior. If $M o e$ specifies $U A I_{\mathbf{t}}^{\text {Moe }}$ for an IAS, then every agent $\mathbf{a}_{i}$ has to comply with this decision by keeping $U A I_{\mathrm{t}}^{\mathbf{a}_{i}}$ to this desired UAI level. However, if there is no UAI imposed by Moe, i.e., $U A I_{\mathbf{t}}^{\mathrm{Moe}}=0$, or if agents are operating at $U A I_{\mathrm{t}}^{\mathrm{IAS}}=0$, then each agent $\mathbf{a}_{\boldsymbol{i}}$ is allowed to operate at its base UAI, denoted by BaseU $A I^{\mathbf{a}_{i}}$. At this UAI level, if required, an agent $\mathbf{a}_{i}$ can use each $\mathbf{c}_{j}$ at $\operatorname{MaxCap_{\mathbf {c}_{j}}} \mathbf{a}_{\boldsymbol{i}}$ and therefore can induce maximum uncertainty dictated by its culture into the system. Hence, the base UAI of an agent dictates the lowest UAI level up to which this agent can operate.

The UAI tuning can be achieved using a utility-based roleexchange technique. A role exchange at any time $\mathrm{t}$ between two IAS agents will swap their current roles at time $\mathbf{t}+\mathbf{1}$. For example, if at time $\mathbf{t}$ an agent $\mathbf{a}_{1}$ is performing role $\mathbf{r}_{1}$ and $\mathbf{a}_{\mathbf{2}}$ is performing role $\mathbf{r}_{2}$, and if they initiate a role exchange, then at time $\mathbf{t}+\mathbf{1}$, agent $\mathbf{a}_{1}$ will perform role $\mathbf{r}_{2}$, whereas $\mathbf{a}_{2}$ will perform role $\mathbf{r}_{1}$. This role exchange will also change the $U A I_{\mathbf{t}}^{\mathbf{a}_{1}}$ and $U A I_{\mathrm{t}}^{\mathbf{a}_{2}}$ according to (2).

Supposing that Moe specifies $U A I_{\mathbf{t}}^{\mathrm{Moe}}$ to an IAS at time $\mathbf{t}$, which is different than $U A I_{\mathbf{t}}^{\mathrm{IAS}}$, then role exchanges are required. Each role exchange requires two agents: roleexchange-seeker agent-agent that seeks a role to exchange, and role-exchange-helper agent-agent that helps the seeker agent by volunteering his role. If a role exchange between agents $a_{s}$ and $a_{h}$ changes, either increase or decrease, the $U A I_{\mathbf{t}}^{\mathbf{a}_{\mathbf{s}}}$ such that $U A I_{\mathbf{t}}^{\mathbf{a}_{\mathbf{s}}}=U A I_{\mathbf{t}}^{\mathbf{M o e}}$, then agents $\mathbf{a}_{\mathbf{s}}$ and $\mathbf{a}_{\mathbf{h}}$ are role-exchange-seeker and role-exchange-helper agents, respectively. When a role exchange is necessary to maintain $U A I_{\mathbf{t}}^{\mathrm{Moe}}$, then each agent $\mathbf{a}_{\mathbf{s}}$ forms a PL, denoted by $P L_{\mathrm{t}}^{\mathbf{a}_{\mathbf{s}}}$, that represents a collection of agents $\mathbf{a}_{\mathbf{h}}$. Let us consider the following two scenarios where role exchange is required.

a) If at time $\mathbf{t} U A I_{\mathbf{t}}^{\mathrm{Moe}}>U A I_{\mathbf{t}}^{\mathrm{IAS}}$, then $M o e$ is trying to increase the UAI of an IAS. In this case, an agent $\mathbf{a}_{\mathbf{s}}$ that is operating below $U A I_{\mathbf{t}}^{\mathrm{Moe}}$, i.e., $U A I_{\mathbf{t}}^{\mathbf{a}_{\mathbf{s}}}<U A I_{\mathbf{t}}^{\mathrm{Moe}}$, becomes a role-exchange-seeker agent to increase its UAI level, and an agent $\mathbf{a}_{\mathbf{h}}$ with $U A I_{\mathbf{t}}^{\mathbf{a}_{\mathbf{h}}} \geq U A I_{\mathbf{t}}^{\text {Moe }}$ becomes a role-exchange-helper agent $\mathbf{a}_{\mathbf{h}}$. Hence, $P L_{\mathbf{t}}^{\mathbf{a}_{\mathbf{s}}}$ is given by

$$
P L_{\mathbf{t}}^{\mathbf{a}_{\mathbf{s}}}=\left\{\mathbf{a}_{\mathbf{h}} \mid U A I_{\mathbf{t}}^{\mathbf{a}_{\mathbf{h}}} \geq U A I_{\mathbf{t}}^{\text {Moe }}\right\} .
$$

b) If at time $\mathbf{t} U A I_{\mathbf{t}}^{\mathrm{Moe}}<U A I_{\mathbf{t}}^{\mathrm{IAS}}$, then $M o e$ is trying to decrease the UAI of an IAS. In this case, an agent $\mathbf{a}_{\mathbf{s}}$ with base UAI that is less than $U A I_{\mathbf{t}}^{\mathrm{Moe}}$, i.e., BaseUAI ${ }^{\mathbf{a}_{\mathrm{s}}}<$ $U A I_{\mathbf{t}}^{\mathrm{Moe}}$, becomes a role-exchange-seeker agent to decrease its UAI level and forms $P L_{\mathbf{t}}^{\mathbf{a}_{\mathbf{s}}}$, given by (3b), with the following role-exchange-helper agents.

i) An agent $\mathbf{a}_{\mathbf{h}}$ with $B a s e U A I^{\mathbf{a}_{\mathbf{h}}} \geq U A I_{\mathbf{t}}^{\mathrm{IAS}}$.

ii) An agent $\mathbf{a}_{\mathbf{h}}$ with base UAI that is greater than or equal to $U A I_{\mathbf{t}}^{\mathrm{Moe}}$ but less than $U A I_{\mathbf{t}}^{\mathrm{IAS}}$, i.e., $U A I_{\mathbf{t}}^{\mathrm{Moe}} \leq$ Base $U A I^{\mathbf{a}_{\mathbf{h}}}<U A I_{\mathbf{t}}^{\mathbf{I A S}}$, becomes a role-exchangehelper agent after resetting its UAI to base UAI, i.e., $U A I_{\mathbf{t}}^{\mathbf{a}_{\mathbf{h}}}=$ BaseUAI ${ }^{\mathbf{a}_{\mathbf{h}}}$

$$
\begin{aligned}
P L_{\mathbf{t}}^{\mathbf{a}_{\mathbf{s}}}=\{ & \left\{\mathbf{a}_{\mathbf{h}} \mid B a s e U A I^{\mathbf{a}_{\mathbf{h}}} \geq U A I_{\mathbf{t}}^{\mathbf{I A S}}\right. \\
& \left.\quad \text { or } U A I_{\mathbf{t}}^{\mathbf{M o e}} \leq B a s e U A I^{\mathbf{a}_{\mathbf{h}}}<U A I_{\mathbf{t}}^{\mathbf{I A S}}\right\} .
\end{aligned}
$$

4) Joint Benefit in IAS: Each role-exchange-seeker agent $\mathbf{a}_{\mathbf{s}}$ has the list of role-exchange-helper agents $\mathbf{a}_{\mathbf{h}}$ in the form of $P L_{\mathbf{t}}^{\mathbf{a}_{\mathbf{s}}}$ calculated either by (3a) or (3b). Now, $\mathbf{a}_{\mathbf{s}}$ has to choose its role-exchange partner from this list. To reach this decision, agent $\mathbf{a}_{\mathbf{s}}$ will calculate the Joint Benefit given by (1) for every agent $\mathbf{a}_{\mathbf{h}}$ in the $P L_{\mathrm{t}}^{\mathbf{a}_{\mathbf{s}}}$. After this calculation, an agent $\mathbf{a}_{\mathbf{h}}$ with the maximum Joint Benefit will be selected as the roleexchange partner of an agent $\mathbf{a}_{\mathbf{s}}$. Calculating Joint Benefit for every agent in the $P L_{\mathbf{t}}^{\mathbf{a}_{\mathbf{s}}}$ exemplifies the rational negotiation strategy, whereas selecting an agent $\mathbf{a}_{\mathbf{h}}$ with maximum Joint Benefit as a role-exchange partner exemplifies the individual optimal role-exchange scheme. Joint Benefit is calculated by computing individual gain, individual loss, social gain, and social loss. Each of these parameters is explained next with respect to IAS.

Individual loss for a role-exchange agent pair $\mathbf{a}_{\mathbf{s}}-\mathbf{a}_{\mathbf{h}}$, denoted by IndLoss $\mathbf{a}_{\mathbf{a}_{s}, \mathbf{a}_{\mathbf{h}}}$, is the measure of capability sacrifice required by the role exchange. The capability sacrifice is the wastage of available resources or capabilities of agents $\mathbf{a}_{\mathbf{s}}$ and $\mathbf{a}_{\mathbf{h}}$ incurred due to performing the exchanged roles. The resources of an agent get wasted if performing a role requires its capabilities at a lesser value than its current role. Hence, calculating individual loss helps us in identifying a role-exchange agent pair with the least resource wastage.

IndLoss $\mathbf{a}_{\mathbf{s}}, \mathbf{a}_{\mathbf{h}}$ is calculated by first computing the future value of each capability for agents $\mathbf{a}_{\mathbf{s}}$ and $\mathbf{a}_{\mathbf{h}}$, i.e., capability usage while performing the exchanged roles, and then by calculating the difference between the current values and the computed future values. The summation of this difference for each capability $\mathbf{c}_{j}$ will give IndLoss $\mathbf{a}_{\mathbf{a}_{s}, \mathbf{a}_{\mathbf{h}}}$. The future value of a capability $\mathbf{c}_{j}$ for an agent $\mathbf{a}_{\boldsymbol{i}}$ at time $\mathbf{t}+\mathbf{1}$ is denoted by 
$\mathbf{v}_{\mathbf{c}_{j}, \mathbf{t}+\mathbf{1}}^{\mathbf{a}_{i}}$. It is equal to either the required value of $\mathbf{c}_{j}$ by a role $\mathbf{r}_{k}$ at time $\mathbf{t}$, i.e., $\mathbf{v}_{\mathbf{c}_{j}, \mathbf{t}}^{\mathbf{r}_{k}}$, or the maximum value of capability $\mathbf{c}_{j}$ that the $C R U_{\mathbf{c}_{j}}^{\mathbf{a}_{i}}$ can generate for agent $\mathbf{a}_{i}$, i.e., $\operatorname{Max} C a p_{\mathbf{c}_{j}}^{\mathbf{a}_{i}}$, whichever is smaller. This is because, as we discussed earlier, an agent can provide a capability value up to the maximum limit which the agent's CRU can generate irrespective of the role's requirements. Hence, if at time $\mathbf{t}$, agent $\mathbf{a}_{\mathbf{s}}$ and $\mathbf{a}_{\mathbf{h}}$ are performing the roles $\mathbf{r}_{1}$ and $\mathbf{r}_{2}$, respectively, then at time $\mathbf{t}+\mathbf{1}$, the future values of a capability $\mathbf{c}_{j}$ for the exchanged roles $\mathbf{r}_{2}$ of agent $\mathbf{a}_{\mathrm{s}}$ and the exchanged roles $\mathbf{r}_{1}$ of agent $\mathbf{a}_{\mathbf{h}}$ are given by (4) and (5), respectively

$$
\begin{aligned}
& \mathbf{v}_{\mathbf{c}_{j}, \mathbf{t}+\mathbf{1}}^{\mathbf{a}_{\boldsymbol{s}}}=\operatorname{Minimum}\left(\mathbf{v}_{\mathbf{c}_{j}, \mathbf{t}}^{\mathbf{r}_{2}}, \operatorname{Max} C a p_{\mathbf{c}_{j}}^{\mathbf{a}_{s}}\right) \\
& \mathbf{v}_{\mathbf{c}_{j}, \mathbf{t + 1}}^{\mathbf{a}_{h}}=\operatorname{Minimum}\left(\mathbf{v}_{\mathbf{c}_{j}, \mathbf{t}}^{\mathbf{r}_{1}}, \operatorname{Max} C a p_{\mathbf{c}_{j}}^{\mathbf{a}_{\boldsymbol{h}}}\right) \text {. }
\end{aligned}
$$

Next, the difference between the future and current values of each capability $\mathbf{c}_{j}$ is calculated. The summation of these differences for each capability $\mathbf{c}_{j}$ multiplied with the capability weight of $\mathbf{c}_{j}$ gives the IndLoss $\mathbf{a}_{\mathbf{s}}, \mathbf{a}_{\mathbf{h}}$, as shown in (6). The capability weight of a capability $\mathbf{c}_{j}$ for an agent $\mathbf{a}_{i}$, i.e., Weight $\mathbf{c}_{\mathbf{c}_{j}}$, is the preference or importance of $\mathbf{c}_{j}$ in the role $\mathrm{r}_{k}$, which agent $\mathbf{a}_{i}$ is performing. Considering every individual capability $\mathbf{c}_{j}$ of the agents and considering the preference of $\mathbf{c}_{j}$ in the form of capability weight preserve and present the additive and lexicographic attributes, respectively, of our jointbenefit utility. However, we will not consider the loss due to a decrease in the value of ULD capabilities in the individual loss calculation because this will be taken into account by social gain, which is discussed later in this section

$$
\begin{aligned}
& \operatorname{IndLoss~}_{\mathbf{a}_{\mathbf{s}}, \mathbf{a}_{\mathbf{h}}}=\sum_{\mathbf{c}_{j} \in \mathbf{C A P}-\mathbf{U C A P}}\left(W e i g h t_{\mathbf{c}_{j}}^{\mathbf{a}_{\mathbf{s}}}\left(\mathbf{v}_{\mathbf{c}_{j}, \mathbf{t}}^{\mathbf{a}_{\mathbf{s}}}-\mathbf{v}_{\mathbf{c}_{j}, \mathbf{t}+\mathbf{1}}^{\mathbf{a}_{\mathbf{s}}}\right)\right. \\
& \left.+W e i g h t_{\mathbf{c}_{j}}^{\mathbf{a h}_{\mathbf{h}}}\left(\mathbf{v}_{\mathbf{c}_{j}, \mathbf{t}}^{\mathbf{a}_{\mathrm{h}}}-\mathbf{v}_{\mathbf{c}_{j}, \mathbf{t}+\mathbf{1}}^{\mathbf{a}_{\mathrm{h}}}\right)\right) .
\end{aligned}
$$

Individual gain for a role-exchange agent pair $\mathbf{a}_{\mathbf{s}}-\mathbf{a}_{\mathbf{h}}$, denoted by $\operatorname{IndGain}_{\mathbf{a}_{\mathrm{s}}, \mathbf{a}_{\mathrm{h}}}$, is the measure of the personal benefit that an agent $\mathbf{a}_{\mathbf{s}}$ receives due to a role exchange. In an ISR operation, a decrease in the damage of a $C R U_{\mathbf{c}_{j}}^{\mathbf{a}_{i}}$ can be considered as a personal benefit to agent $\mathbf{a}_{\boldsymbol{i}}$. In our experiments, the decrease in a CRU damage is calculated as the difference between the damage to the $C R U_{\mathbf{c}_{j}}^{\mathbf{a}_{\mathbf{s}}}$ at time $\mathbf{t}$ while performing a role $\mathbf{r}_{\boldsymbol{k}}$ at $U A I_{\mathbf{t}}^{\mathbf{I A S}}$, i.e., $C R U \operatorname{Damage} e_{\mathbf{c}_{j}, \mathbf{t}}^{\mathbf{a}_{\mathbf{s}}}\left(U A I_{\mathbf{t}}^{\mathbf{I A S}}\right)$, and the average (or arithmetic mean) of damages to the $C R U_{\mathbf{c}_{j}}^{\mathbf{a}_{\mathbf{s}}}$ at time $\mathbf{t}^{\prime}$ while performing the same role at $U A I_{\mathbf{t}}^{\mathrm{a}_{\mathrm{h}}}$, i.e., AvgCRUDamage $\mathbf{c}_{\mathbf{c}_{i}, \mathbf{t}^{\prime}}^{\mathbf{a}_{\mathbf{s}}}\left(U A I_{\mathbf{t}}^{\mathbf{I A S}}\right)$. Time $\mathbf{t}^{\prime}$ represents all the previous instances of ISR operations, which are similar to the current operation. Hence, it gives the reference to the past experience in similar condition. The summation of these benefits for all the CRUs of the agent $\mathbf{a}_{\mathbf{s}}$ will give us the $\operatorname{IndGain}_{\mathbf{a}_{\mathbf{s}}, \mathbf{a}_{\mathbf{h}}}$, as shown in (7). Ideally, the IndGain $\mathbf{a}_{\mathbf{s}}, \mathbf{a}_{\mathbf{h}}$ will be higher when $\mathbf{a}_{\mathbf{h}}$ belongs to an agent culture with high UAI level because, at a high UAI level, an agent $\mathbf{a}_{\mathbf{s}}$ will behave more stealthily and hence incur less damage due to less severe attacks and the counterattacks with the enemy units

$$
\begin{aligned}
\operatorname{IndGain}_{\mathbf{a}_{\mathbf{s}}, \mathbf{a}_{\mathbf{h}}}= & \sum_{\mathbf{c}_{\mathbf{j}} \in \mathbf{C A P}}\left(C R U \operatorname{Damag} e_{\mathbf{c}_{\mathbf{j}}, \mathbf{t}}^{\mathbf{a}_{\mathbf{s}}}\left(U A I_{\mathbf{t}}^{\mathbf{I A S}}\right)\right. \\
& \left.-\operatorname{AvgCRUD\operatorname {Damag}e_{\mathbf {c}_{\mathbf {j}},\mathbf {t}}^{\mathbf {a}_{\mathbf {s}}}}\left(U A I_{\mathbf{t}}^{\mathbf{a}_{\mathbf{h}}}\right)\right) .
\end{aligned}
$$

Social gain for a role-exchange agent pair $\mathbf{a}_{\mathbf{s}}-\mathbf{a}_{\mathbf{h}}$, denoted by SocialGain $_{\mathbf{a}_{\mathbf{s}}, \mathbf{a}_{\mathbf{h}}}$, is the measure of social benefit that an IAS receives due to the role exchange. Social gain is calculated with reference to the maximal social gain, which a human supervisor defines as one of the goals during a mission planning. For example, during an ISR mission planning, Moe can dictate that performing ISR on all the target locations at $U A I_{\mathbf{t}}^{\mathrm{Mooe}}$ will give maximal social gain or MaximalSocialGain ${ }^{\text {Moe }}$. Moe also defines a social gain reduction or SocialGainReduction ${ }^{\mathrm{Moe}}$ that represents a per unit value by which the social gain would reduce if an agent $\mathbf{a}_{\mathbf{s}}$ opts a role exchange with agent $\mathbf{a}_{\mathbf{h}}$, where $U A I_{\mathrm{t}}^{\mathrm{a}_{\mathrm{h}}}$ is not exactly equal to $U A I_{\mathrm{t}}^{\mathrm{Moe}}$. Hence, the absolute difference between $U A I_{\mathbf{t}}^{\mathrm{a}_{\mathbf{h}}}$ and $U A I_{\mathbf{t}}^{\mathrm{Moe}}$ gives the factor by which SocialGainReduction ${ }^{\mathrm{Moe}}$ is deducted from MaximalSocialGain ${ }^{\text {Moe }}$. Equation (8) shows how SocialGain $\mathbf{a}_{\mathbf{s}}, \mathbf{a}_{\mathbf{h}}$ can be calculated using the maximal social gain and social gain reduction. Based on this equation, we can say that, the higher the closeness of UAI level of agent $\mathbf{a}_{\mathbf{h}}$ to the $U A I_{\mathbf{t}}^{\text {Moe }}$ value, the larger the value of SocialGain $\mathbf{a}_{\mathbf{s}}, \mathbf{a}_{\mathbf{h}}$

$$
\begin{aligned}
& \text { SocialGain }_{\mathbf{a}_{\mathbf{s}}, \mathbf{a}_{\mathbf{h}}}=\text { MaximalSocialGain }^{\text {Moe }} \\
& \quad-\left(\left|U A I_{\mathbf{t}}^{\mathrm{Moe}}-U A I_{\mathbf{t}}^{\mathbf{a}_{\mathbf{h}}}\right|\right) \text { SocialGainReduction } \\
& \text { Moe }
\end{aligned}
$$

Lastly, social loss for a role-exchange agent pair $\mathbf{a}_{\mathbf{s}}-\mathbf{a}_{\mathbf{h}}$, denoted by SocialLoss $\mathbf{a}_{\mathbf{s}}, \mathbf{a}_{\mathbf{h}}$, is the measure of loss to the IAS incurred due to agent $\mathbf{a}_{\mathbf{h}}$ leaving its current role unfinished due to the role exchange with agent $\mathbf{a}_{\mathbf{s}}$. Leaving a role unfinished may require rollback of some completed parts of a role, which leads to a hidden loss to an IAS.

\section{IDV and PDI Inclusion in IAS}

Incorporating IDV and PDI in our algorithm helped us magnify the effect of these cultural parameters on the overall benefit of the approach. Moreover, IDV and PDI appear together in a culture; therefore, experiment with one parameter will automatically reveal other parameter's effect on an IAS.

NCW emphasizes on making ISR components (or agents) collectivist [16]. Such collectivist agents will be very sensitive to the demand of their social context and more responsible to assumed the needs of others, as well as less insistent on pursuing personal goals that might jeopardize group benefits [16]. Also, collectivist agents similar to collectivist people are more likely to attribute their own and others behavior to situational rather than dispositional causes [11]. We can easily visualize the Pareto optimality nature in such collectivist culture agents.

The reason for including PDI in our research is to study the effect of hierarchical relationship or inequality, which is inevitable in a work setting where superior-subordinate relationships exist. According to Cummings et al., the human supervisory control in UAV operation is hierarchical in nature [2]. Although we have not made these agents hierarchical with respect to each other in our approach, we did introduce hierarchy using agent-supervisor relation. Hence, by including this parameter, we could be able to see the effect of inequality, which is always certain while working at Sheridan's fifth and 
sixth automation levels [21]. We discussed in Section II that the agents at high PDI (or low IDV) consider supervisor as a benevolent dictator and hence obey her directives.

\section{IMPLEMENTATION AND EXPERIMENTS}

The collaboration of heterogeneous entities in ISR operations is customary. This leads to the scenarios where different components become responsible for different tasks, working toward a common goal. Our implementation of role-exchange algorithm replicates the nature of collaboration most commonly found in ISR. A simulation is designed and fully implemented using programming language $\mathrm{C}$ and OpenGL, which is used to generate data that illustrate several metrics. We have presented UVs as agents. In this section, we begin by explaining the nature of agent capabilities. Next, the details of simulator scenarios are offered.

\section{A. Capabilities and Their ULD Nature}

As discussed before, we used the capabilities of the ISR agents as the main attribute that distinguishes among agents in our approach. An agent can have any number and any type of capabilities. To make our experiments tractable and to mimic a battlefield scenario, we have used four most basic military operations, namely, attack $(\mathbf{A})$, patrol $(\mathbf{P})$, reconnaissance $(\mathbf{R})$, and communicate $(\mathbf{C})$, as capabilities available to an ISR agent.

Attack (A) in the warfare is mandatory for any manned or unmanned combat-capable ISR. A direct inevitable consequence of attack is ambiguity and unfamiliarity. Hence, with increase in $\mathbf{A}$, uncertainty increases or UAI decreases in an IAS. Therefore, $\mathbf{A}$ is a member of ULD capability set UCAP, i.e., $\mathbf{A} \in \mathbf{U C A P}$. Equation (9) represents the relationship of $\mathrm{A}$ and UAI

$$
\mathbf{A} \propto \frac{1}{U A I} .
$$

Patrol $(\mathbf{P})$ and reconnaissance $(\mathbf{R})$ capabilities are also indispensable for any ISR operation and play vital roles. However, they generally do not lead to uncertainty. Therefore, $\mathbf{P}$ and $\mathbf{R}$ capabilities are non-ULD capabilities. Lastly, we have considered communicate $(\mathbf{C})$ capability, which exemplifies the communicative nature of an ISR agent. Using $\mathbf{C}$, battlefield awareness gets increased as it helps to share information about the adversary's activities and current status of available resources. This information will alleviate the state of confusion and ambiguity among peer group members and aid in the decision-making process of fellow ISR agents. This implies that, with increase in $\mathbf{C}$, uncertainty decreases or UAI increases, as shown in

$$
\mathbf{C} \propto U A I .
$$

Hence, based on (10) and (11), the relationship between $\mathbf{C}$ and UAI is opposite to that of $\mathbf{A}$ and UAI or we can say that the inverse of $\mathbf{C}$, i.e., $\mathbf{C}^{\prime}$, is directly proportional to $\mathbf{A}$, as given by

$$
\mathbf{C}^{\prime} \propto \mathbf{A} .
$$

Understanding the relation among the ULD capabilities is very important. Let us consider a situation where Moe wants to control the current UAI level of an IAS. Controlling several parameters requires that the direction of all the parameters is conjugate. If two ULD capabilities are growing in the reverse direction, for example, capabilities $\mathbf{A}$ and $\mathbf{C}$, it is very difficult to increase or decrease both of them simultaneously using a single control tool. According to the proposed definition of supervisory control discussed in Section III, the more appropriate supervisory control would be the one that removes the micromanagement of trivial tasks. However, to keep track of several control tools at a same time will lead to micromanagement. Hence, selecting $\mathbf{C}^{\prime}$ instead of $\mathbf{C}$ will avoid such a situation and allows Moe to control these two ULD capabilities using a single control parameter. Moreover, the presence of $\mathbf{A}$ and $\mathbf{C}^{\prime}$ at the same time also allows us to examine the effect of having more than one ULD capabilities in the ISR system.

\section{B. Battle Space Simulation}

For the simulation of our approach, we considered the three cultural parameters of MOTL, namely, UAI, IDV, and PDI, in a cumulative fashion and explored their impact and interrelationships in the ISR context. Our simulator also studies each parameter with respect to two different IASs, namely, 3cap and $4 c a p$. A 3 cap IAS has three capabilities, whereas a 4 cap IAS has four.

A screenshot of our simulator is shown in Fig. 3. For simulation purposes, we assumed a territory of interest that spreads from the point $\mathbf{X}$ to the point $\mathbf{Y}$ in the simulated battlefield and is required to be explored using ISR. Several enemy units are also depicted in the simulated battlefield in the form of diamond-shape icons. The IAS agents are presented in the form of rectangular boxes. The intensity of the color of these boxes represents their current UAI levels; the darker shades symbolize agents with higher current UAI. The simulator also provides a control for Moe to explicitly tune the UAI level at run time using the command line. Hence, this control allows him to adjust $U A I_{\mathbf{t}}^{\mathrm{IAS}}$ by specifying $U A I_{\mathbf{t}}^{\mathrm{Moe}}$ at random points multiple times during an ISR operation. Using this control, an operator can act upon any changing or exigent situation by imposing a UAI to the IAS, which is traversing the territory of interest.

However, the screenshot shown in Fig. 3 presents a scenario when $U A I_{\mathbf{t}}^{\text {Moe }}=U A I_{\mathbf{t}}^{\text {IAS }}=0$, i.e., no UAI is imposed over IAS, hence no role exchange would take place while traversing the territory of interest. Therefore, each agent $\mathbf{a}_{i}$ will operate on its BaseU $A I^{\mathbf{a}_{i}}$ and incur a high total damage. The group loss field in the screenshot is showing the reset value of zero, which should not be confused with no group loss. We can also observe a cleared region along the convoy's path in the territory of interest in this screenshot. This area represents the fully destroyed enemy forces by the attack of IAS agents. The encircled diamonds symbolize the damaged enemy forces that were attacked but not fully destroyed. The attacking incurs a total damage or total CRU damage of 10.5 units to the 3 cap IAS. This damage is the summation of the damages sustained by all the CRUs in the IAS due to the adversary's 




Fig. 3. Snapshot of simulator battlefield for 3cap IAS.

TABLE I

3capIAS

\begin{tabular}{|c|c|c|c|c|c|}
\hline $\begin{array}{c}\text { Agent } \\
\left(\mathbf{a}_{i}\right)\end{array}$ & $\begin{array}{c}\text { Initial } \\
\text { Role } \\
\left(\mathbf{r}_{k}\right)\end{array}$ & $\begin{array}{c}\text { Maximum } \\
\text { Attack } \\
\text { Capability } \\
\left(\text { MaxCap }_{A}^{a_{i}}\right)\end{array}$ & $\begin{array}{c}\text { Maximum } \\
\text { Patrol } \\
\text { Capability } \\
{\text { (MaxCap } \mathbf{a}_{\mathrm{p}} \text { ) }}^{\mathbf{M}^{2}}\end{array}$ & $\begin{array}{c}\text { Maximum } \\
\text { Reconnaissance } \\
\text { Capability } \\
\left(\text { MaxCap }_{\mathrm{R}}\right)\end{array}$ & $\begin{array}{c}\text { Base UAI } \\
\text { Level } \\
\left.\text { (BaseUAI }^{\mathbf{a}_{i}}\right)\end{array}$ \\
\hline$\overline{\mathbf{A}}$ & $\overline{\mathbf{A}}$ & 100 & - & - & 0 \\
\hline $\begin{array}{l}\text { AR } \\
\text { AP }\end{array}$ & $\begin{array}{l}\text { AR } \\
\text { AP }\end{array}$ & $\begin{array}{l}60 \\
60\end{array}$ & $\begin{array}{c}- \\
40\end{array}$ & $\begin{array}{l}40 \\
-\end{array}$ & 1 \\
\hline $\begin{array}{l}\text { ARP } \\
\text { APR } \\
\end{array}$ & $\begin{array}{l}\text { ARP } \\
\text { APR } \\
\end{array}$ & $\begin{array}{l}50 \\
50 \\
\end{array}$ & $\begin{array}{l}20 \\
30\end{array}$ & $\begin{array}{l}30 \\
20\end{array}$ & 2 \\
\hline $\begin{array}{l}\text { RA } \\
\text { PA }\end{array}$ & $\begin{array}{l}\text { RA } \\
\text { PA }\end{array}$ & $\begin{array}{l}40 \\
40\end{array}$ & $\begin{array}{l}- \\
60\end{array}$ & $\begin{array}{l}60 \\
-\end{array}$ & 3 \\
\hline $\begin{array}{l}\text { RAP } \\
\text { PAR }\end{array}$ & $\begin{array}{l}\text { RAP } \\
\text { PAR }\end{array}$ & $\begin{array}{l}30 \\
30\end{array}$ & $\begin{array}{l}20 \\
50\end{array}$ & $\begin{array}{l}50 \\
20\end{array}$ & 4 \\
\hline $\begin{array}{l}\text { RPA } \\
\text { PRA } \\
\end{array}$ & $\begin{array}{l}\text { RPA } \\
\text { PRA } \\
\end{array}$ & $\begin{array}{l}20 \\
20 \\
\end{array}$ & $\begin{array}{l}30 \\
50\end{array}$ & $\begin{array}{l}50 \\
30\end{array}$ & 5 \\
\hline $\begin{array}{c}\mathbf{R} \\
\mathbf{P} \\
\mathbf{R P} \\
\mathbf{P R}\end{array}$ & $\begin{array}{c}\mathbf{R} \\
\mathbf{P} \\
\mathbf{R P} \\
\mathbf{P R}\end{array}$ & $\begin{array}{l}- \\
- \\
-\end{array}$ & $\begin{array}{c}- \\
100 \\
40 \\
60\end{array}$ & $\begin{array}{c}100 \\
- \\
60 \\
40\end{array}$ & 6 \\
\hline
\end{tabular}

counteractions. The total CRU damage for 4cap ISR is even higher, i.e., 13.7 units (not shown in the figure) because there are more agents per UAI-level-defined cultures.

In our experiments, the agents and roles are named or labeled using the combination of the capabilities $\mathbf{A}, \mathbf{P}, \mathbf{R}$, and $\mathbf{C}^{\prime}$. For example, if a role $\mathbf{r}_{k}$ requires the capabilities $\mathbf{c}_{1}, \mathbf{c}_{2}, \ldots, \mathbf{c}_{n}$ at values $\mathbf{v}_{\mathbf{c}_{1}, \mathbf{t}}^{\mathbf{r}_{k}}, \mathbf{v}_{\mathbf{c}_{2}, \mathbf{t}}^{\mathbf{r}_{k}}, \ldots, \mathbf{v}_{\mathbf{c}_{n}, \mathbf{t}}^{\mathbf{r}_{k}}$, respectively, and $\mathbf{v}_{\mathbf{c}_{1}, \mathbf{t}}^{\mathbf{r}_{k}} \geq \mathbf{v}_{\mathbf{c}_{2}, \mathbf{t}}^{\mathbf{r}_{k}} \geq \cdots \geq \mathbf{v}_{\mathbf{c}_{n}, \mathbf{t}}$, then $\mathbf{r}_{k}$ is labeled using capabilities such that the capabilities are arranged in the descending order of the values. Hence, the role label of $\mathrm{r}_{k}$ would be $\mathbf{c}_{1}, \mathbf{c}_{2}, \ldots, \mathbf{c}_{\boldsymbol{n}}$. In addition, if $\mathbf{v}_{\mathbf{c}_{j}, \mathbf{t}}^{\mathbf{r}_{\boldsymbol{k}}}=0$, then $\mathbf{c}_{j}$ will not be a part of a role's label. Initially, each of these labeled roles is assigned to an agent $\mathbf{a}_{\boldsymbol{i}}$ such that, for every capability $\mathbf{c}_{j}$, $\mathbf{v}_{\mathbf{c}_{j}, \mathrm{t}}^{\mathbf{a}_{i}}=\mathbf{v}_{\mathbf{c}_{j}, \mathrm{t}}^{\mathbf{r}_{k}}$. Moreover, if we use the same naming convention for labeling the agents, then the functional label of the $\mathbf{a}_{i}$ will exactly be same as that of the label of $\mathbf{r}_{k}$. The roles, agents, and capabilities available in 3cap and 4cap IASs are discussed next, which will clearly present this naming convention technique.

1) 3cap IAS: In a 3cap IAS, capabilities $\mathbf{A}, \mathbf{P}$, and $\mathbf{R}$ were used. Hence, $\mathbf{C A P}=\{\mathbf{A}, \mathbf{P}, \mathbf{R}\}$ and $\mathbf{U C A P}=\{\mathbf{A}\}$. The total number of capability combinations, taken one, two, and three capabilities at a time from three capabilities, is ${ }^{3} \mathrm{P}_{1}+{ }^{3} \mathrm{P}_{2}+{ }^{3} \mathrm{P}_{3}=15$. Hence, 15 agents and 15 roles are initialized using the naming convention discussed earlier. Table I shows the agents along with their initially assigned roles and $\operatorname{MaxCap_{\mathbf {c}_{j}}} \mathbf{a}_{\boldsymbol{i}}$ for each capability $\mathbf{c}_{j}$. BaseU $A I^{\mathbf{a}_{i}}$ is also shown in the table, which is calculated using (2). The 15 agents are divided into seven agent cultures using BaseU $A I^{\mathbf{a}_{i}}$, each of which represents a different UAI level. 
TABLE II

COMPUTATION OF IndLoss $\mathbf{A R}, \mathbf{a}_{\mathbf{h}}$ WHEN $U A I_{\mathbf{t}}^{\mathbf{I A S}}=0$ AND $U A I_{\mathbf{t}}^{\text {Moe }}=3$

\begin{tabular}{|c|c|c|c|c|c|c|c|c|c|c|c|c|c|c|c|}
\hline & $\mathrm{A}$ & $\mathrm{R}$ & - & $\mathrm{R}$ & A & - & & & A & $\mathrm{R}$ & - & $P$ & A & - & \\
\hline MaxCap & 60 & 40 & - & 60 & 40 & - & & Max Cap & 60 & 40 & - & 60 & 40 & - & \\
\hline CurrentCap & 60 & 40 & - & 60 & 40 & - & & CurrentCap & 60 & 40 & - & 60 & 40 & - & \\
\hline FutureCap & 40 & 40 & - & 40 & 40 & - & & FutureCap & 40 & 0 & - & 0 & 40 & - & \\
\hline \multirow[t]{2}{*}{ IndLoss } & - & 0 & 0 & 12 & 0 & 0 & 12 & IndLoss & - & 16 & 0 & 36 & 0 & \begin{tabular}{l|l}
0 & 5 \\
\end{tabular} & 52 \\
\hline & $\mathrm{A}$ & $\mathrm{R}$ & - & $\mathrm{R}$ & $\mathrm{A}$ & $P$ & & & $\mathrm{~A}$ & $\mathrm{R}$ & - & $P$ & $\mathrm{~A}$ & $\mathrm{R}$ & \\
\hline MaxCap & 60 & 40 & - & 50 & 30 & 20 & & MaxCap & 60 & 40 & - & 50 & 30 & 20 & \\
\hline CurrentCap & 60 & 40 & - & 50 & 30 & 20 & & CurrentCap & 60 & 40 & - & 50 & 30 & 20 & \\
\hline FutureCap & 30 & 40 & - & 40 & 30 & 0 & & FutureCap & 30 & 20 & - & 0 & 30 & 20 & \\
\hline \multirow[t]{2}{*}{ IndLoss } & - & 0 & 0 & 5 & 0 & 4 & 09 & IndLoss & - & 8 & 0 & 25 & 0 & 0 & 33 \\
\hline & 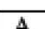 & & 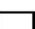 & $\mathrm{BP}$ & $P$ & A & & & & & & & & & \\
\hline MaxCap &  & $\pi$ & - & 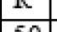 & $\mathrm{F}$ & A & & & A & $\mathrm{R}$ & - & $P$ & $\mathrm{~K}$ & A & \\
\hline & 60 & 40 & - & 50 & 30 & 20 & & Max Cap & 60 & 40 & - & 50 & 30 & 20 & \\
\hline CurrentCap & 60 & 40 & - & 50 & 30 & 20 & & CurrentCop & 60 & 40 & - & 50 & 30 & 20 & \\
\hline FutureCap & 20 & 40 & - & 40 & 0 & 20 & & FutureCap & 20 & 30 & - & 0 & 30 & 20 & \\
\hline \multirow[t]{3}{*}{ IndLoss } & - & 0 & 0 & 5 & 9 & 0 & 14 & IndLoss & - & 4 & 0 & 25 & 0 & 0 & 29 \\
\hline & & & & & & & & & & & & & & & \\
\hline & A & $\mathrm{R}$ & - & $\mathrm{R}$ & - & - & & & A & $\mathrm{R}$ & - & $P$ & - & - & \\
\hline Max Cap & 60 & 40 & - & 100 & - & - & & Max Cap & 60 & 40 & - & 100 & 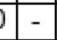 & - & \\
\hline CurrentCap & 60 & 40 & - & 100 & - & - & & CurrentCap & 60 & 40 & - & 100 & 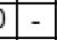 & - & \\
\hline FutureCap & 0 & 40 & - & 40 & - & - & & FutureCap & 0 & 0 & - & 0 & - & - & \\
\hline IndLoss & - & 0 & 0 & 60 & 0 & 0 & 60 & IndLoss & - & 16 & 0 & 100 & \begin{tabular}{|l|l|}
0 & 0 \\
\end{tabular} & 0 & 116 \\
\hline & & & & & & & & & & & & & & & \\
\hline & $\mathrm{A}$ & $\mathrm{R}$ & - & $\mathrm{R}$ & $P$ & - & & & $\mathrm{A}$ & $\mathrm{R}$ & - & $P$ & $\mathrm{R}$ & - & \\
\hline MaxCap & 60 & 40 & - & 60 & 40 & - & & MaxCap & 60 & 40 & - & 60 & 40 & - & \\
\hline CurrentCap & 60 & 40 & - & 60 & 40 & $1-$ & & CurrentCap & 60 & 40 & - & 60 & 40 & - & \\
\hline Future Cap & 0 & 40 & - & 40 & 0 & - & & FutureCap & 0 & 40 & - & 0 & 40 & - & \\
\hline IndLoss & - & 0 & 0 & 12 & 16 & 0 & 18 & IndLoss & - & 0 & 0 & 36 & 0 & 0 & 36 \\
\hline
\end{tabular}

Initially, when $U A I_{\mathrm{t}}^{\mathrm{Moe}}$ is also zero, each agent $\mathbf{a}_{i}$ performs a role $\mathbf{r}_{k}$ using $\mathbf{c}_{j}$ at $\mathbf{v}_{\mathbf{c}_{j}, \mathbf{t}}^{\mathbf{a}_{i}}=\operatorname{Max} \operatorname{Cap} p_{\mathbf{c}_{j}}^{\mathbf{a}_{i}}$, and the agent culture with the lowest UAI value, i.e., $U A I_{\mathbf{t}}^{\mathbf{A}}=0$, dictates the $U A I_{\mathbf{t}}^{\mathrm{IAS}}=0$. Furthermore, to mathematically elucidate the calculations in the experiments, for each $\mathbf{c}_{j}$, an agent $\mathbf{a}_{\boldsymbol{i}}$ is initialized with the capability value $\mathbf{v}_{\mathbf{c}_{j}, \mathbf{t}}^{\mathbf{a}_{i}}$ such that their summation $\mathbf{v}_{\mathbf{c}_{1}, \mathbf{t}}^{\mathbf{a}_{i}}+\mathbf{v}_{\mathbf{c}_{2}, \mathbf{t}}^{\mathbf{a}_{\mathbf{i}}}+\cdots+\mathbf{v}_{\mathbf{c}_{n}, \mathbf{t}}^{\mathbf{a}_{i}}=100$.

2) 4cap IAS: In a 4cap IAS, in addition to $\mathbf{A}, \mathbf{P}$, and $\mathbf{R}$, capability $\mathbf{C}^{\prime}$ is also used. Hence, $\mathbf{C A P}=\left\{\mathbf{A}, \mathbf{P}, \mathbf{R}, \mathbf{C}^{\prime}\right\}$, and $\mathbf{U C A P}=\left\{\mathbf{A}, \mathbf{C}^{\prime}\right\}$. The total number of capability combinations is 64 in this case. Hence, 64 agents and 64 roles are initialized using the same naming convention. For each agent, BaseU AI ${ }^{\mathbf{a}_{\boldsymbol{i}}}$ was calculated using (2). Based upon these values, the 64 agents are divided into nine agent cultures, each of which represents a different UAI level. The main reason to consider such a larger scenario is to study and reveal the impact of the presence of more than one ULD capabilities in an IAS. In the 4 cap IAS, $50 \%$ of the agent's capabilities are ULD in comparison with $33 \%$ of the 3 cap IAS. Moreover, the difference in ULD capabilities' preferences can also exist in an IAS, which might lead to other significant consequences. However, in our experiments with 4cap IAS, we gave the same preference to both ULD capabilities.

To discuss the calculation involved in steps 3 and 4 of our algorithm (Fig. 2), let us consider the UAI tuning in both directions: low-to-high and high-to-low UAI levels.

3) Tuning From Low UAI to High UAI Level: In order to change the $U A I_{\mathbf{t}}^{\mathrm{IAS}}$ of an IAS, Moe can impose a $U A I_{\mathbf{t}}^{\mathrm{Moe}}$ on an IAS. For instance, at time $\mathbf{t}$ when $U A I_{\mathbf{t}}^{\mathrm{IAS}}=0$, if Moe specifies $U A I_{\mathbf{t}}^{\mathrm{Moe}}=3$, then according to the roleexchange algorithm discussed, few agents have to change their UAI levels to this desired value. Since $U A I_{\mathbf{t}}^{\text {Moe }}>$ $U A I_{\mathbf{t}}^{\mathbf{I A S}}$, each agent $\mathbf{a}_{\mathbf{s}}$ with $U A I_{\mathbf{t}}^{\mathbf{a}_{\mathbf{s}}}<3$ would become a role-exchange-seeker agent, whereas every agent $\mathbf{a}_{\mathbf{h}}$ with $U A I_{\mathbf{t}}^{\mathbf{a}_{\mathbf{h}}} \geq 3$ would be a role-exchange-helper agent. Hence, in a 3 cap IAS, agents at zero, one, and two UAI levels, namely, agent $\mathbf{A}, \mathbf{A R}, \mathbf{A P}, \mathbf{A R P}$, and $\mathbf{A P R}$, are agents $\mathrm{a}_{\mathrm{s}}$. The $P L_{\mathrm{t}}^{\mathrm{a}_{\mathrm{s}}}$ will be computed for each of these agents, using (3a). For example, the PL of the agent AR, which is performing a role $\mathbf{A R}$, at time $\mathbf{t}$ will contain the helper agents at UAI levels three, four, five, and six, i.e., $P L_{\mathbf{t}}^{\mathbf{A R}}=$ $\{$ RA, PA, RAP, PAR, RPA, PRA, P, R, RP, PR $\}$. After identifying $\mathbf{a}_{\mathbf{s}}$ and $\mathbf{a}_{\mathbf{h}}$ agents, the next step is to calculate the Joint Benefit, which requires individual loss, individual gain, social gain, and social loss to be calculated first.

For example, let us consider the individual loss calculation for agent $\mathbf{A R}$ or IndLoss $\mathbf{A R}_{\mathbf{R}, \mathbf{a}_{\mathbf{h}}}$ of a 3cap IAS, where $\mathbf{a}_{\mathbf{h}}$ represents a helper agent in the $P L_{\mathbf{t}}^{\mathbf{A R}}$. The calculation of IndLoss $_{\mathbf{A R}, \mathbf{a}_{\mathbf{h}}}$ is shown is Table II. The ten individual losses calculated using (6), one for each agent $\mathbf{a}_{\mathbf{h}}$, are shown in a separate subtable of Table II. In the subtables, MaxCap represents

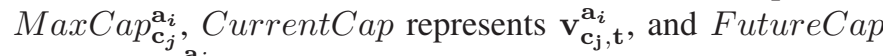
represents $\mathbf{v}_{\mathbf{c}_{\mathbf{j}}, \mathbf{t}+\mathbf{1}}$.

For instance, let us look at the calculation performed to obtain the IndLoss AR,RAP. First, the FutureCap (or $\left.\mathbf{v}_{\mathbf{c}_{j}, \mathbf{t}+\mathbf{1}}^{\mathbf{a}_{i}}\right)$ is calculated for all the capabilities of agent $\mathbf{A R}$ and 
RAP using MaxCap (or MaxCap $\mathbf{c}_{j}^{\mathbf{a}_{i}}$ ) and CurrentCap (or $\mathbf{v}_{\mathbf{c}_{j}, \mathbf{t}}^{\mathbf{a}_{i}}$ ). As $U A I_{\mathbf{t}}^{\mathbf{I A S}}=0$, therefore, $\mathbf{v}_{\mathbf{c}_{j}, \mathbf{t}}^{\mathbf{a}_{i}}=\operatorname{Max} C a p_{\mathbf{c}_{j}}^{\mathbf{a}_{i}}$. Hence, for agent $\mathbf{A R}$, the $\operatorname{Max} \operatorname{Cap}_{\mathbf{A}}^{\mathbf{A R}}=\mathbf{v}_{\mathbf{A}, \mathbf{t}}^{\mathbf{A R}}=60$, $\operatorname{Max} \operatorname{Cap}_{\mathbf{P}}^{\mathbf{A R}}=\mathbf{v}_{\mathbf{P}, \mathbf{t}}^{\mathbf{A R}}=$ null (because capability $\mathbf{P}$ is not available with agent $\mathbf{A R}$ ), and $\operatorname{Max} C a p_{\mathbf{R}}^{\mathbf{R A P}}=\mathbf{v}_{\mathbf{R}, \mathbf{T}}^{\mathbf{R A P}}=40$. Similarly, for agent RAP, $\operatorname{Max} C a p_{\mathbf{A}}^{\mathbf{R A P}}=\mathbf{v}_{\mathbf{A}, \mathbf{t}}^{\mathbf{R A P}}=30$, $\operatorname{Max} C a p_{\mathbf{P}}^{\mathbf{R A P}}=\mathbf{v}_{\mathbf{P}, \mathbf{t}}^{\mathbf{R A P}}=20, \quad$ and $\quad \operatorname{Max} C a p_{\mathbf{P}}^{\mathbf{R A P}}=$ $\mathbf{v}_{\mathbf{P}, \mathbf{t}}^{\mathbf{R A P}}=50$. The FutureCap calculation of capability $\mathbf{R}$ for both the agents using (4) and (5) is performed as

$$
\begin{aligned}
\mathbf{v}_{\mathbf{R}, \mathbf{t}+\mathbf{1}}^{\mathbf{A R}} & =\operatorname{Minimum}\left(\mathbf{v}_{\mathbf{R}, \mathbf{t}}^{\mathbf{R A P}}, \operatorname{Max} \operatorname{Map}_{\mathbf{R}}^{\mathbf{A R}}\right) \\
& =\operatorname{Minimum}(50,40) \\
& =40 \\
\mathbf{v}_{\mathbf{R}, \mathbf{t}+\mathbf{1}}^{\mathbf{R A P}} & =\operatorname{Minimum}\left(\mathbf{v}_{\mathbf{R}, \mathbf{t}}^{\mathbf{A R}}, \operatorname{Max} C a p_{\mathbf{R}}^{\mathbf{R A P}}\right) \\
& =\operatorname{Minimum}(40,50) \\
& =40 .
\end{aligned}
$$

After calculating the remaining FutureCap in similar fashion, the IndLoss AR,RAP is calculated using FutureCap and weight of all the capabilities as the second step. In our experiments, the weight of a capability in a role is calculated with respect to its required value in that role. The bigger the required value of a capability $\mathbf{c}_{j}$ in a role $\mathbf{r}_{k}$, i.e., $\mathbf{v}_{\mathbf{c}_{j}, \mathbf{t}}^{\mathbf{r}_{k}}$, the higher the value of Weight $t_{\mathbf{c}_{j}}^{\mathbf{a}_{j}}$. We have used $1 \%$ of the $\mathbf{v}_{\mathbf{c}_{j}, \mathbf{t}}$ as $W e i g h t_{\mathbf{c}_{j}}^{\mathbf{r}_{\mathbf{s}}}$. For example, the weights of capabilities $\mathbf{R}, \mathbf{A}$, and $\mathbf{P}$ for agent RAP performing role RAP are $0.5,0.3$, and 0.2 , respectively. However, according to (6), the ULD capabilities of the roleexchange-seeker agent are not considered in the individual loss calculation. The calculation for IndLoss AR, RAP using (6) is performed as

$$
\begin{aligned}
\text { IndLoss }_{\mathbf{A R}, \mathbf{R A P}}= & \left(\text { Weight } \mathbf{R}_{\mathbf{R}}^{\mathbf{R}}\left(\mathbf{v}_{\mathbf{R}, \mathbf{t}}^{\mathbf{A R}}-\mathbf{v}_{\mathbf{R}, \mathbf{t}+\mathbf{1}}^{\mathbf{A R}}\right)\right) \\
& +\left(\text { Weight } \mathrm{R}_{\mathbf{R}}^{\mathbf{R A P}}\left(\mathbf{v}_{\mathbf{R}, \mathbf{t}}^{\mathbf{R A P}}-\mathbf{v}_{\mathbf{R}, \mathbf{t}+\mathbf{1}}^{\mathbf{R A P}}\right)\right) \\
& +\left(\text { Weight } \mathbf{A}_{\mathbf{A}}^{\mathbf{R A P}}\left(\mathbf{v}_{\mathbf{A}, \mathbf{t}}^{\mathbf{R A P}}-\mathbf{v}_{\mathbf{A}, \mathbf{t}+\mathbf{1}}^{\mathbf{R A P}}\right)\right) \\
& +\left(\text { Weight } \mathbf{P}_{\mathbf{P}}^{\mathbf{R A P}}\left(\mathbf{v}_{\mathbf{P}, \mathbf{t}}^{\mathbf{R A P}}-\mathbf{v}_{\mathbf{P}, \mathbf{t}+\mathbf{1}}^{\mathbf{R A P}}\right)\right) \\
= & (0.4(40-40))+(0.5(50-40)) \\
& +(0.3(30-30))+(0.2(20-0)) \\
= & 0+5+0+4=9 .
\end{aligned}
$$

The value of IndLoss AR,RAP, i.e. nine units, is minimum in all the individual losses shown in Table II. Therefore, if agent AR exchanges role with agent RAP, then the individual loss would be least. However, the remaining three components of the joint benefit will also influence the agent AR's final partner selection.

The individual gain or the personal benefit of the same agent AR due to the role exchange with a

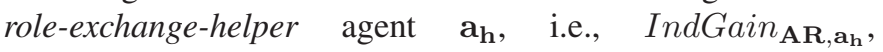
is calculated using (7). For example, if $\mathbf{c}_{j}=\mathbf{R}$ and $\mathbf{a}_{\mathbf{h}}=\mathbf{R A P}, \quad$ then $\quad C R U$ Damage $\mathbf{R}_{\mathbf{R}, \mathbf{t}}^{\mathbf{A R}}\left(U A I_{\mathbf{t}}^{\mathbf{I A S}}\right)-$ $A v g C R U D a m a g e_{\mathbf{R}, \mathbf{t}^{\prime}}^{\mathbf{A R}}\left(U A I_{\mathbf{t}}^{\mathbf{I A S}}\right)$ gives the decrease in the damage of $C R U_{R}^{\mathrm{AR}}$ due to the role exchange with agent RAP. The decrease in the damage of other CRUs of agent pair AR - RAP will also be calculated in similar fashion. The summation of all these values would give us IndGain AR,RAP.

The SocialGain $_{\mathbf{A R}, \mathbf{a}_{\mathbf{h}}}$ is calculated using (8). In our experiment, IAS performing ISR from point $\mathbf{X}$ to point $\mathbf{Y}$ at $U A I_{\mathbf{t}}^{\mathrm{Moe}}$ is considered as the MaximalSocialGain ${ }^{\text {Moe }}$. This implies that SocialGain $_{\mathbf{A R , R A}}$ and SocialGain $\mathbf{A R , P A}$ will be maximum or equal to MaximalSocialGain ${ }^{\mathrm{Moe}}$. Also, the social gain reduces by the largest factor or to minimum if agent AR exchanges role with any one of the four agents at UAI level 6 or $U A I_{\mathbf{t}}^{\mathbf{a}_{\mathbf{h}}}=6$.

Finally, for SocialLoss $\mathbf{A R}, \mathbf{a}_{\mathbf{h}}$, we used randomly generated values to mimic the percentage of a role that is already performed by agent $\mathbf{a}_{\mathbf{h}}$ when it is asked for the role exchange with agent AR. However, if we keep IDV and PDI inactivated, then the social loss would be less influential toward the final selection of partners.

4) Tuning from High UAI to Low UAI Level: Moe can also decrease the UAI of an IAS during an ISR operation. Let us consider an intermediate instance, when $U A I_{\mathrm{t}}^{\mathrm{IAS}}=5$ and Moe specifies $U A I_{\mathbf{t}}^{\mathrm{Moe}}=2$. Since $U A I_{\mathbf{t}}^{\mathrm{Moe}}<U A I_{\mathbf{t}}^{\mathrm{IAS}}$, each agent $\mathbf{a}_{\mathbf{s}}$ with BaseU $A I^{\mathbf{a}_{\mathbf{s}}}<U A I_{\mathbf{t}}^{\mathrm{Moe}}$ would become a roleexchange-seeker agent. Therefore, agents at UAI levels 0 and 1 or agent $\mathbf{A}, \mathbf{A R}$, and $\mathbf{A P}$ would become role-exchangeseeker agents $\mathbf{a}_{\mathbf{s}}$ 's. To identify the role-exchange-helper agents, let us consider the agent AP that is performing the role RPA at time t. According to (3b), the $P L_{\mathbf{t}}^{\mathbf{A P}}$ contains 12 agents: ARP, APR, RA, PA, RAP, PAR, RPA, PRA, P, $\mathbf{R}, \mathbf{R P}$, and $\mathbf{P R}$. Agents with $B a s e U A I^{\mathrm{a}_{\mathbf{h}}} \geq U A I_{\mathbf{t}}^{\mathrm{IAS}}$, i.e., agents at UAI levels 5 and 6 , would be included into $P L_{\mathbf{t}}^{\mathrm{AP}}$ as they are. Therefore, CurrentCap (or $\mathbf{v}_{\mathbf{c}_{j}, \mathbf{t}}^{\mathbf{a}_{\mathbf{h}}}$ ) for these agents is need not be equal to $\operatorname{Max} C a p$ (or $\operatorname{Max} C a p_{\mathbf{c}_{j}}^{\mathbf{a}_{\mathbf{h}}}$ ). The agents at UAI levels 2, 3, and 4 for which $U A I_{\mathbf{t}}^{\text {Moe }} \leq$ BaseUAI $I^{\mathrm{a}_{\mathrm{h}}}<$ $U A I_{\mathbf{t}}^{\text {IAS }}$ will be included to $P L_{\mathbf{t}}^{\mathbf{A P}}$ after resetting there UAI to base UAI; therefore, CurrentCap is equal to MaxCap for these agents. The joint benefit will be calculated similar to the previous example.

\section{RESUltS AND CONCLUSION}

This section presents the results of the experiments discussed in Section IV as the validation of our approach. Generated data are plotted as graphs for two scenarios. The first scenario depicts the UAI influence on the CRU damage of 3 cap and 4 cap IASs, whereas in the second scenario, the UAI-IDV influence on Average IndGain of 3cap IAS is presented.

\section{A. UAI Influence on IAS}

The adversary's counteractions on IAS agents while traversing the territory of interest have damaged the CRUs of IAS. The summation of the damages sustained by all CRUs of each agent in an IAS is called the total CRU damage or TotalCRUDamage ${ }^{\mathrm{IAS}}$. We used our simulator to illustrate the effect of different UAI levels of 3cap and 4cap IASs on TotalCRUDamage ${ }^{\mathrm{IAS}}$. The range of UAI levels for 3 cap 


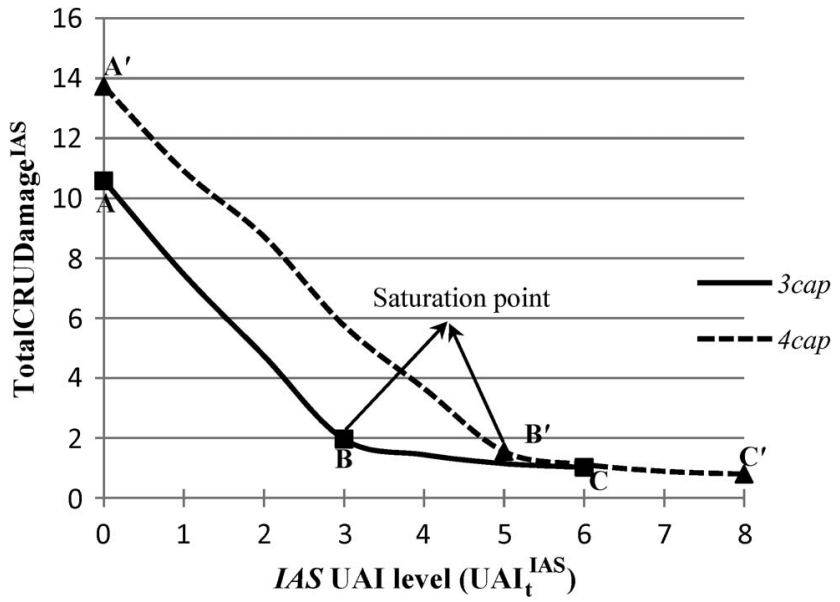

Fig. 4. TotalCRU Damage $\mathrm{IAS}^{\mathrm{IA}}$ at different UAI levels for 3 cap and 4 cap IASs.

IAS is zero to six, whereas it is zero to eight for 4cap IAS. In Fig. 4, the curves $A B C$ and $A^{\prime} B^{\prime} C^{\prime}$ represent the total $C R U$ damages incurred to 3cap and 4cap IASs, respectively, at different UAI levels.

TotalCRUDamage $\mathrm{IAS}^{\mathrm{IAS}}$ values for both 3 cap and 4 cap IASs are maximum at $U A I_{\mathbf{t}}^{\mathrm{IAS}}=0$, as shown by points $A$ and $A^{\prime}$ in the graph. Since no UAI is enforced on IASs at this level, therefore, every agent is performing its role using $\operatorname{Max} \operatorname{Cap}_{\mathbf{c}_{j}} \mathbf{a}_{i}$. We can also observe that the TotalCRUDamage $e^{4 c a p}>$ TotalCRUDamage ${ }^{3 c a p}$ for all the UAI values. This difference is due to the difference in the numbers of agents and the difference in the numbers of ULD capabilities in two IASs. Therefore, we can conclude that, the larger the group of agents, the less stealthy it can keep its moves and, similarly, the larger number of ULD capabilities are difficult to control.

In spite of these differences between the two IASs, the gradual decrease in their CRU damage can be observed with an increase in $U A I_{\mathbf{t}}^{\mathrm{IAS}}$. This shows the benefit of using our approach. The decrease is very sharp for 3 cap IAS from point $A$ to point $B$, i.e., from CRU damage of 10.3 units at $U A I_{\mathbf{t}}^{\text {IAS }}=0$ to 2 units at $U A I_{\mathbf{t}}^{\mathrm{IAS}}=3$, but the changes in UAI value from point $B$ to $C$ do not lead to significant benefits. Similarly, the decrease in CRU damage became almost saturated after point $\mathrm{B}^{\prime}$ for 4 cap IAS. Hence, $U A I_{\mathbf{t}}^{\mathrm{IAS}}=3$ and $U A I_{\mathbf{t}}^{\mathrm{IAS}}=5$ are the saturation points of the UAI values for $3 c a p$ and 4cap IASs, respectively, beyond which the CRU damage did not decreased significantly.

\section{B. IDV Influence on IAS}

In order to illustrate the effect of IDV on the IAS, we have augmented IDV with UAI and have examined its influence on the individual gain of agents in an IAS. We considered three different $U A I_{\mathbf{t}}^{\text {Moe }}$, namely, 1, 3, and 6, for 3cap IAS, which represent the UAI spectra at lowest, median, and highest values, respectively. We computed the effect of IDV values that range from -5 to +5 with each of these three UAI levels on the individual gain. The IAS at $I D V=-5$ represents a collectivist IAS that possesses agents with a highest collectivist belief, whereas $I D V=+5$ represents an individualistic IAS where agents possess a highest individualistic belief.

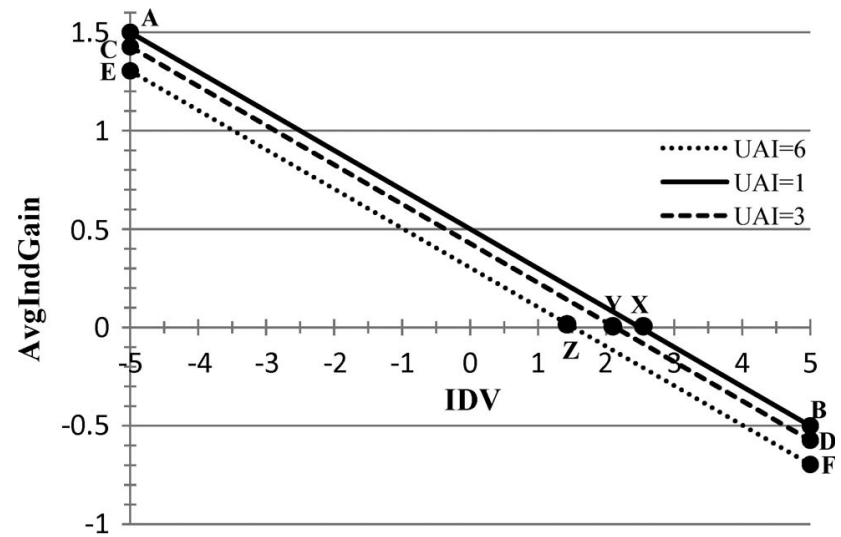

Fig. 5. AvgIndGain versus $I D V$ for 3cap IAS.

First, the maximum value of IndGain $\mathbf{a}_{\mathbf{s}}, \mathbf{a}_{\mathbf{h}}$ is identified for every role-exchange-seeking agent $\mathbf{a}_{\mathbf{s}}$ at a particular $U A I_{\mathbf{t}}^{\mathrm{Moe}}$ and an IDV, and then, the arithmetic mean of these individual gain values or AvgIndGain is computed. Fig. 5 shows the AvgIndGain of a 3 cap IAS at different values of IDV for three different $U A I_{\mathbf{t}}^{\mathbf{I A S}}$ values. The line segments $A B, C D$, and $E F$ represent AvgIndGain at $U A I_{\mathbf{t}}^{\mathbf{I A S}}: 1,3$ and 6, respectively. The AvgIndGain at $I D V=-5$ for all the three $U A I_{\mathbf{t}}^{\mathrm{Moe}}$ values, shown as points $A, C$, and $E$, is maximum. However, with the increase in IDV value, it decreased and even became negative after points $X, Y$, and $Z$. Since the role-exchangehelper agents in a collectivist IAS implicitly possess high PDI that makes them strictly obey the $U A I_{\mathbf{t}}^{\mathrm{Moe}}$, therefore, a higher individual gain is attained as compared to individualistic IAS, where the low PDI allows agents to work in a more unrestrained manner. This dictates that the agents in a collectivist IAS generate more positive individual gain than the agents in an individualistic IAS.

We can also observe that the AvgIndGain is less for higher $U A I_{\mathbf{t}}^{\mathrm{Moe}}$ because a high $U A I_{\mathrm{t}}^{\mathrm{Moe}}$ leads to more numbers of role-exchange-seeker agents in an IAS and hence reduces the AvgIndGain due to greater competition among these agents for the most beneficial role-exchange-helper agents. For instance, at $U A I_{\mathbf{t}}^{\mathrm{Moe}}=1$ in 3 cap IAS, there was only one $\mathbf{a}_{\mathbf{s}}$ agent. Hence, without any contender, the role exchange occurs with an agent that leads to maximum IndGain $\operatorname{Ias}_{\mathbf{a}_{\mathbf{s}}, \mathbf{a}_{\mathbf{h}}}$. On the contrary, when $U A I_{\mathbf{t}}^{\mathrm{Moe}}=3$ and $U A I_{\mathbf{t}}^{\mathrm{Moe}}=6$, the numbers of $\mathbf{a}_{\mathrm{s}}$ agents were 5 and 11 , respectively, which lead to a race condition and keep agents away from the most benefited matches for their role exchange.

\section{FUTURE WORK}

Reported research can be extended in many directions. For instance, a power-based hierarchy of agents can be introduced on the top of UAI and IDV in an agent society. This would merge the advantages of MOTL with other proposed hierarchical control models [2]. Furthermore, the hierarchical division of agents would also allow us to establish supervisor-subordinate relationship among agents. In such an agent society, a supervisor agent would control or manage its subordinate agents on the commands and directions of human supervisor. 
Implementing such a supervisor-subordinate agent relationship using MOTL would lead us to another futuristic concept that we can call "agent on the loop" (AOTL). AOTL will be useful to engender a simplified supervisory control for envisioned fully automated multiplatform cooperative UV swarms.

Similarly, the IDV can be more deeply explored in a platform diverse agent community. This would reveal the consequences when a member of "newcomer" agents or out-group agents interacts with the member of "historical" or in-group agents in an agent society [1]. Multiplatform software coordination is an example of such interaction, where culturally alike group of software, which is a pivotal part that forms in-group-interacts with many culturally dissimilar out-group software for a collective goal attainment in a cooperative task.

Lastly, the priority given to the two ULD capabilities A and $\mathbf{C}^{\prime}$ was equal in this paper; however, ULD capabilities with different priorities can also be studied. An extensive exploration of complex decision-making systems, such as defense or aerospace systems, would unleash other ULD and nonULD capabilities with their relative importance and hence help materializing real-world MOTL-based supervisory control.

\section{REFERENCES}

[1] S. Bochner and B. Hesketh, "Power distance, individualism/collectivism, and job-related attitudes in a culturally diverse work group," J. Cross-Cult. Psychol., vol. 25, no. 2, pp. 233-257, 1994.

[2] M. L. Cummings, S. Bruni, S. Mercier, and P. J. Mitchell, "Automation architecture for single operator, multiple UAV Command and Control," Int. Command Control J., vol. 1, no. 2, pp. 1-24, 2007.

[3] R. M. Cyert and J. G. March, A Behavioral Theory of the Firm. Englewood Cliffs, NJ: Prentice-Hall, 1963.

[4] Network Centric Warfare: Department of Defense Report to Congress. Washington, DC: OSD, U.S. Dept. Defense, 2001.

[5] The Implementation of Network-Centric Warfare. Washington, DC: Office Force Transformation, OSD, Dept. Defense, 2003.

[6] Unmanned Aircraft Systems (UAS) Roadmap, 2005-2030. Washington, DC: OSD, Dept. Defense, 2005.

[7] P. C. Fishburn, "Utility theory," Manage. Sci., vol. 14, no. 5, pp. 335-378, Jan. 1968

[8] H. Hexmoor, B. McLaughlan, and G. Tuli, "Towards a natural human role in supervising complex control systems," J. Exp. Theor. Artif. Intell., vol. 21, no. 1, pp. 59-77, Mar. 2009.

[9] G. Hofstede, Culture's Consequences: International Differences in Work-Related Values. Beverly Hills, CA: Sage, 1980.

[10] G. Hofstede, Culture's Consequences: Comparing Values, Behaviors, Institutions and Organizations Across Nations. Thousand Oaks, CA: Sage, 2001.

[11] J. Jaspers and M. Hewstone, "Cross-cultural interaction, social attributions and inter-group relations," in Cultures in Contact: Studies in CrossCultural Interaction. Oxford, U.K.: Pergamon, 1982.

[12] S. Kalenka and N. R. Jennings, "Socially responsible decision making by autonomous agents," in Proc. Int. Conf. Complex Syst., 1997, pp. $135-149$.

[13] U. Kim, H. C. Triandis, C. Kaqitcibasi, S. Choi, and G. Yoon, Eds., Individualism and Collectivism: Theory, Method, and Applications, vol. 18. Thousand Oaks, CA: Sage, 1994, ser. Cross-Cultural Research and Methodology Series.
[14] D. Lambert and J. Scholz, "A Dialectic for Network Centric Warfare," in Proc. 10th Int. Command Control Res. Technol. Symp., 2005. [Online]. Available: http://www.dodccrp.org/events/10th_ ICCRTS/CD/papers/ 016.pdf

[15] H. R. Markus and S. Kitayama, "Culture and the self: Implications for cognition, emotion, and motivation," Psychol. Rev., vol. 98, no. 2, pp. 224-253, 1991.

[16] Y. K. Ng, Welfare Economics: Towards a More Complete Analysis. Hampshire, NY: Palgrave MacMillan, 2004, ch. 2.

[17] W. A. Owens, Lifting the Fog of War. Baltimore, MD: Johns Hopkins Univ. Press, 2001.

[18] C. J. Petrie, T. A. Webster, and M. R. Cutkosky, "Using Pareto optimality to coordinate distributed agents," Artif. Intell. Eng., Des., Manuf., vol. 9, no. 4, pp. 269-281, 1995 .

[19] A. Rahman and H. Hexmoor, "Negotiation to improve role adoption in organizations," in Proc. Int. Conf. Artif. Intell., 2004, pp. 476-480.

[20] T. B. Sheridan, Telerobotics, Automation, and Human Supervisory Control. Cambridge, MA: MIT Press, 1992.

[21] T. B. Sheridan and W. L. Verplank, "Human and computer control of undersea teleoperators," MIT Man-Mach. Lab., Cambridge, MA, Tech. Rep., 1978.

[22] J. C. Strassner, Policy-Based Network Management: Solution for the Next Generation. San Francisco, CA: Morgan Kaufmann, 2004.

[23] U.S. Air Force Scientific Advisory Board, Air Force Operations in Urban Environments, vol. 1, Washington, DC, DC: U.S. Air Force2005.

[24] X. Zhang and H. Hexmoor, "Utility based role exchange," in Proc. Central Eastern Eur. Multi-Agent Syst., 2001, pp. 332-340.

[25] X. Zhang and H. Hexmoor, "Algorithms for utility-based role exchange," in Proc. 7th Int. Conf. Intell. Auton. Syst., 2002, pp. 396-403.

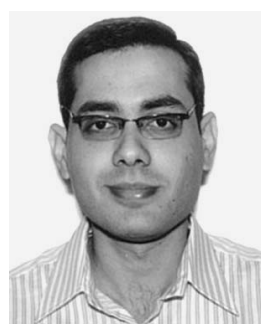

Gaurav Tuli was born in Mehsana, India, on December 12, 1983. He received the B.E. degree in computer engineering from the University of Rajasthan, Jaipur, India, in 2005 and the M.S. degree in computer science from Southern Illinois University, Carbondale, IL, in 2008. He is currently working toward the Ph.D. degree with the Computer Science Department, Virginia Polytechnic Institute and State University, Blacksburg.

He was a Graduate Research Assistant with the Computer Science Department and the Biochemistry and Molecular Biology Department, Southern Illinois University, from January 2007 to December 2007 and from June 2007 to October 2008, respectively. His research interests include multiagent system, artificial intelligence, ontology mediation, and bioinformatics.

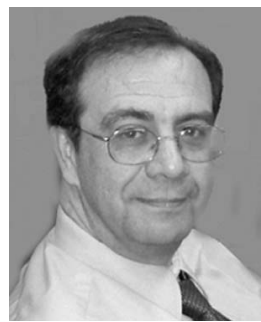

Henry Hexmoor (M'04-SM'05) received the M.S. degree from Georgia Tech, Atlanta, and the Ph.D. degree in computer science from the State University of New York, Buffalo, in 1996.

He taught at the University of North Dakota before a stint at the University of Arkansas. He is currently an Assistant Professor with the Computer Science Department, Southern Illinois University, Carbondale, IL. He has published widely in artificial intelligence and multiagent systems. His research interests include multiagent systems, artificial intelligence, cognitive science, and mobile robotics. 